\title{
44TH ANNUAL SASKATCHEWAN CHRISTMAS MAMMAL COUNT - 2016
}

\section{Alan R. Smith}

Box 154

Avonlea, SK SOH OCO

alanrandi@sasktel.net

Eighty-eight Christmas Mammal Counts were conducted this winter three more than last year. The 3,443 mammals seen or heard were up by 40 per cent over the 2,458 animals recorded in 2015. Over half of this change was, however, a result of an increase in the numbers of Mule Deer, which were up from 490 to 1,005. In comparison, White-tailed Deer were only up from 758 to 856 .

Several high count records were set or tied. Five Northern Flying Squirrels at Togo tied the high count set last year at Greenwater. Estevan set the record for Eastern Cottontail with 15, the previous high was five at Estevan on January 4, 2010 and January 1, 2014. Two American
Marten at Togo on January 5, 2017 set a new high exceeding singles at Squaw Rapid on the 1986 count, Love in 2000, Togo in 2007 and 2008, Prince Albert National Park in 2009, and Christopher Lake in 2012. Bison are flourishing in Grasslands National Park: 237 were counted, tripling the previous high of 77 in 2012.

With 14 species seen or heard, Odessa bested Togo's 11 for the most species seen or heard on a count. For the ninth year, no new species were added this winter so the all-time provincial total remains at 51 species seen or heard (plus three species found dead and two others recorded only on the basis of tracks).

For information on participants, weather, coverage and location of CMCs, see the CBC summary that will be provided in the next issue of Blue Jay.

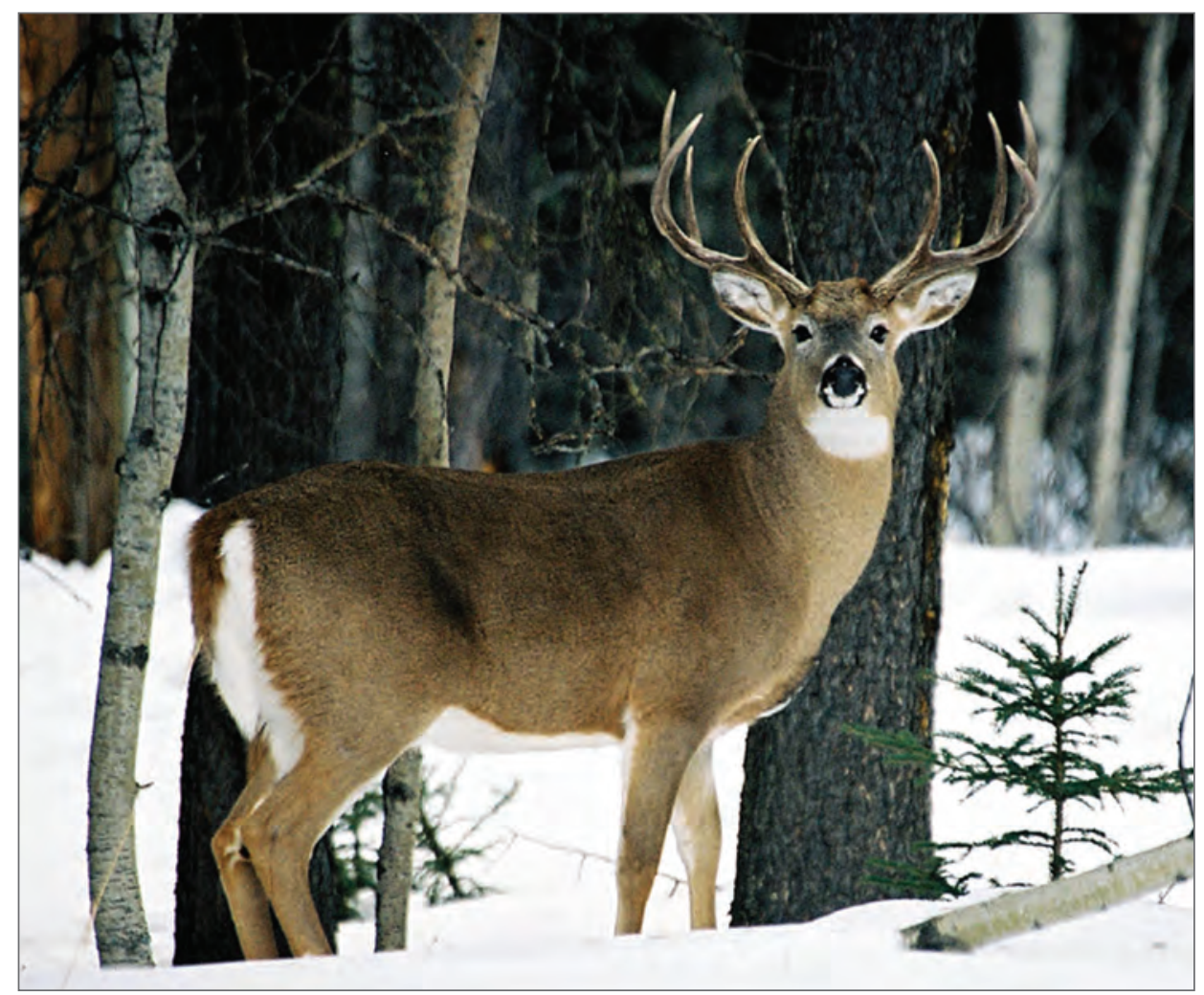

White-tailed Deer. Photo credit: Randy McCulloch

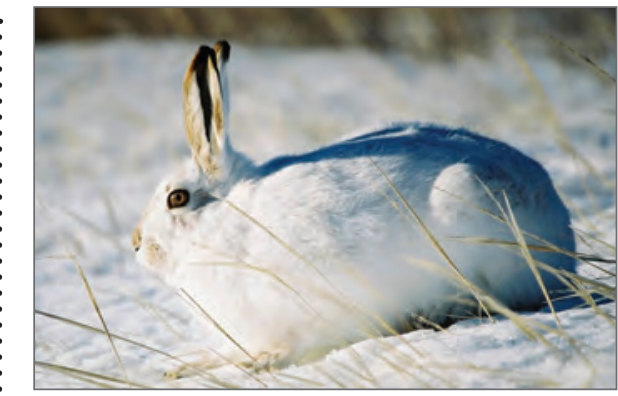

White-tailed Jackrabbit Photo credit: Randy McCulloch

\section{Explanation \\ of entries in Table 1.}

The number of mammals actually seen or heard on count day is treated separately from those recorded by other means, or those recorded during count period (December 14 to January 5) but not on count day. Numbers of individuals seen or heard are given in Table 1 and are tallied in the first line of totals at the bottom of the table. The number of species they represent is given in the second line.

For species only detected by tracks or by other means, or that are seen or heard only in the count period but not on count day, no numbers of individuals is given in Table 1. Species detected only by tracks are indicated by ' $t$ ' in the table; those detected only by other means - dead animals ' $m$ ', more clearly identifiable chewing or digging ' $d$ ', dens or lodges ' $L$ ' (including Muskrat push-ups) and by smell 's.' Species detected by any means during the count period, but not on count day are indicated by ' $c$ ' in the table. These additional species are tallied in lines 3, 4 and 5 at the bottom of the table. If a mammal is reported as member of a species group (i.e. mouse species, deer species), it is counted as a species only if no other species in this group has been definitely recorded. The columns at the end of the table give totals for each species. 


\begin{tabular}{|c|c|c|c|c|c|c|c|c|c|c|c|c|c|c|c|c|c|c|c|c|c|c|c|c|}
\hline SPECIES & 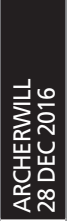 & 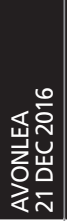 & 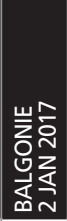 & 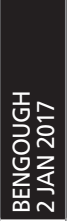 & 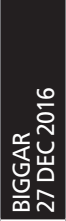 & 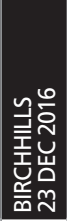 & 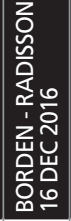 & 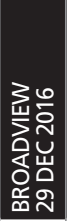 & 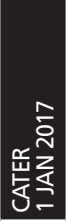 & 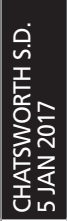 & 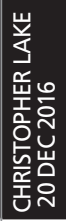 & 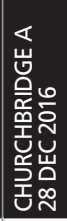 & 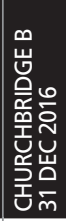 & 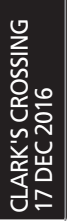 & 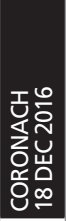 & 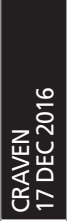 & 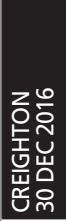 & 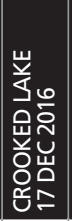 & 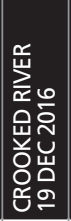 & 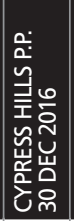 & 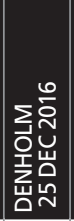 & 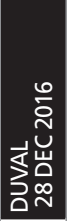 & 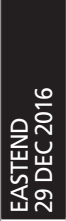 & 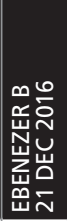 \\
\hline Shrew species & c & & & & & $\mathrm{t}$ & & & & & & & & & & & $\mathrm{t}$ & & & & & & & \\
\hline \multicolumn{25}{|l|}{ Eastern Cottontail } \\
\hline Nuttall's Cottontail & & & & & & & & & & & & & & & & 2 & & & & 1 & & & 4 & \\
\hline Snowshoe Hare & $\mathrm{t}$ & & $\mathrm{t}$ & & $\mathrm{t}$ & $\mathrm{t}$ & & $\mathrm{t}$ & $\mathrm{t}$ & & $\mathrm{t}$ & & & $\mathrm{t}$ & & 1 & $\mathrm{t}$ & & 1 & & 1 & & & 2 \\
\hline White-tailed Jack Rabbit & & & 1 & & $\mathrm{t}$ & & & $\mathrm{t}$ & & & & & & 1 & $\mathrm{t}$ & & & $\mathrm{t}$ & & & & $\mathrm{t}$ & & \\
\hline Eastern Grey Squirrel & & & 2 & & & & & & & & & & & & & 2 & & & & & & & & \\
\hline Eastern Fox Squirrel & & 5 & 16 & 1 & & & & 4 & & & & & & & & 4 & & & & & & 6 & & \\
\hline American Red Squirrel & 6 & & & & & 1 & 2 & 6 & 2 & 4 & 3 & 1 & 2 & & & 4 & 3 & 4 & 1 & 3 & & & & \\
\hline Northern Flying-Squirrel & & & & & & & & & & & & & & & & & & & & & 1 & & & \\
\hline \multicolumn{25}{|l|}{ Northern Pocket Gopher } \\
\hline American Beaver & & & & & & L & & $\mathrm{L}$ & & $\mathrm{L}$ & & & & & & & & & & & & $\mathrm{L}$ & & \\
\hline Deer Mouse & c & & & & & & & $\mathrm{t}$ & & & $\mathrm{t}$ & & & & & & & & & & c & & & \\
\hline Muskrat & & & & & $\mathrm{L}$ & $\mathrm{L}$ & & $\mathrm{L}$ & $\mathrm{L}$ & & & & & & & & & $\mathrm{L}$ & & & c & $\mathrm{L}$ & & \\
\hline Gapper's Red-Backed Vole & & & & & & & & & & & & & & & & & & & & & c & & & \\
\hline Meadow Vole & & & & & & & & & & & 1 & & & & & & & & & & & & & \\
\hline Vole species & & & & & $\mathrm{t}$ & & & & & & & & & & & 1 & $\mathrm{t}$ & & & & & $\mathrm{t}$ & & \\
\hline \multicolumn{25}{|l|}{ House Mouse } \\
\hline Mouse species & & & & & & $\mathrm{t}$ & & & & & & & & & $\mathrm{t}$ & $\mathrm{t}$ & 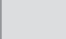 & & 1 & & & $\mathrm{t}$ & & \\
\hline American Porcupine & c & & & & & & 1 & & & & & & & & & & & & & & & & & \\
\hline Coyote & c & & 1 & & 3 & $\mathrm{t}$ & 7 & $\mathrm{t}$ & & 1 & 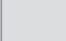 & & 1 & 13 & 8 & 6 & $\mathrm{t}$ & 1 & 1 & & 1 & 1 & 9 & 1 \\
\hline Gray Wolf & & & & & & & & & & & & & & & & & $\mathrm{t}$ & & & & & & & \\
\hline Red Fox & & & $\mathrm{t}$ & & 1 & $\mathrm{t}$ & & $\mathrm{t}$ & & & $\mathrm{t}$ & & & 2 & $\mathrm{t}$ & 1 & 1 & 1 & & & 1 & $\mathrm{t}$ & 1 & \\
\hline Raccoon & $\mathrm{t}$ & & & & & & & & & & & & & & & $\mathrm{m}$ & & & & & & & & \\
\hline American Marten & & & & & & & & & & & 1 & & & & & & & & & & & & & \\
\hline Fisher & & & & & & & & $\mathrm{t}$ & & & & & & & & & & & & & & & & \\
\hline Ermine & & & & & & $\mathrm{t}$ & & $\mathrm{t}$ & & & & & & & & $\mathrm{t}$ & 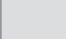 & & & & 1 & $\mathrm{t}$ & & \\
\hline Long-tailed Weasel & & & & & & $\mathrm{t}$ & & $\mathrm{t}$ & & $\mathrm{t}$ & & & & & & & 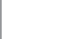 & & & & & $\mathrm{t}$ & 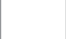 & \\
\hline Weasel species & & & & & & & & & & & & & & & & & $\mathrm{t}$ & & & & & & $\mathrm{t}$ & \\
\hline American Mink & 1 & & 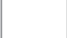 & & 1 & & & & & & & & $\mathrm{t}$ & & $\mathrm{t}$ & & & & & & & & & \\
\hline American Badger & 1 & & $\mathrm{t}$ & & & $\mathrm{t}$ & & & & & & & & & & & & $\mathrm{t}$ & & & & & & 1 \\
\hline \multicolumn{25}{|l|}{ Striped Skunk } \\
\hline River Otter & & & & & & $\mathrm{t}$ & & & & & & & & 1 & & & & & & & & & & \\
\hline Mountain Lion & & & & & & & & $\mathrm{t}$ & & & & & & & & & $\mathrm{t}$ & & & & & & & \\
\hline \multicolumn{25}{|l|}{ Canada Lynx } \\
\hline Mule Deer & & & 38 & 21 & 16 & & 1 & 4 & 1 & & . & & & 12 & 63 & 19 & & 2 & & & c & & 206 & \\
\hline White-tailed Deer & 7 & 7 & 4 & 14 & 9 & & 8 & $\mathrm{t}$ & 4 & 19 & $\mathrm{t}$ & & c & 26 & 4 & 45 & 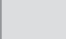 & 7 & 8 & 12 & 7 & 10 & 42 & 2 \\
\hline Deer species & & & & & & $\mathrm{t}$ & & & & & & & & & & & & & & & & & & \\
\hline Moose & 2 & & & & & & 2 & 1 & & & & & c & & & & $\mathrm{t}$ & 1 & & 2 & 1 & c & & 6 \\
\hline Elk & c & & & & & & & & & & & & & & & & & & & & & & & \\
\hline \multicolumn{25}{|l|}{ Pronghorn } \\
\hline \multicolumn{25}{|l|}{ American Bison } \\
\hline $\begin{array}{l}\text { TOTALS SEEN/HEARD } \\
\text { ON COUNT DAY }\end{array}$ & 17 & 12 & 62 & 36 & 30 & 1 & 21 & 15 & 7 & 24 & 5 & 1 & 3 & 55 & 75 & 85 & 4 & 16 & 12 & 18 & 13 & 17 & 262 & 12 \\
\hline TOTAL SPECIES SEEN/HEARD & 5 & 2 & 6 & 3 & 5 & 1 & 6 & 4 & 3 & 3 & 3 & 1 & 2 & 6 & 3 & 10 & 2 & 6 & 5 & 4 & 7 & 3 & 5 & 5 \\
\hline $\begin{array}{l}\text { TOTAL SPECIES } \\
\text { RECORDED BY TRACKS }\end{array}$ & 2 & 0 & 3 & 0 & 3 & 10 & 0 & 10 & 1 & 1 & 4 & 0 & 1 & 1 & 4 & 2 & 9 & 2 & 0 & 0 & 0 & 6 & 1 & 0 \\
\hline $\begin{array}{l}\text { TOTAL SPECIES } \\
\text { OTHERWISE RECORDED }\end{array}$ & 0 & 0 & 0 & 0 & 1 & 2 & 0 & 2 & 1 & 1 & 0 & 0 & 0 & 0 & 0 & 1 & 0 & 1 & 0 & 0 & 0 & 2 & 0 & 0 \\
\hline $\begin{array}{l}\text { SPECIES RECORDED } \\
\text { COUNT PERIOD }\end{array}$ & 5 & 0 & 0 & 0 & 0 & 0 & 0 & 0 & 0 & 0 & 0 & 0 & 2 & 0 & 0 & 0 & 0 & 0 & 0 & 0 & 4 & 1 & 0 & 0 \\
\hline $\begin{array}{l}\text { TOTAL SPECIES } \\
\text { COUNT PERIOD AND DAY }\end{array}$ & 12 & 2 & 9 & 3 & 9 & 13 & 6 & 16 & 5 & 5 & 7 & 1 & 5 & 7 & 7 & 12 & 11 & 9 & 5 & 4 & 11 & 12 & 6 & 5 \\
\hline
\end{tabular}




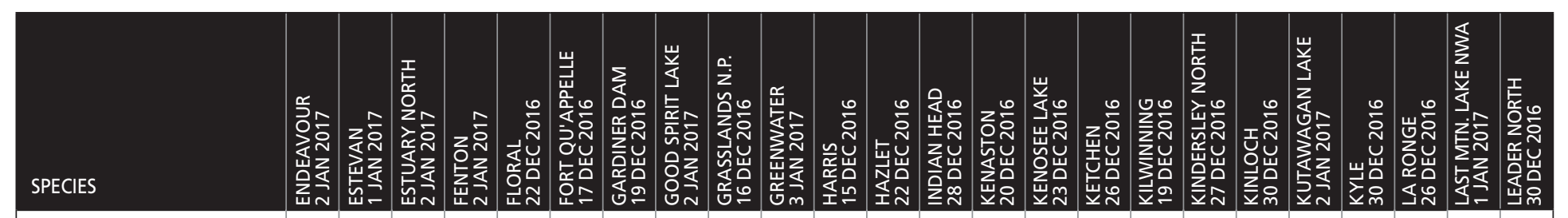

Shrew species

Eastern Cottontail

Nuttall's Cottontail

15

Snowshoe Hare

White-tailed Jack Rabbit

Eastern Grey Squirrel

Eastern Fox Squirrel

American Red Squirrel

Northern Flying-Squirrel

Northern Pocket Gopher

American Beaver

Deer Mouse

Muskrat

Gapper's Red-Backed Vole

Meadow Vole

Vole species

House Mouse

Mouse species

American Porcupine

Coyote

Gray Wolf

Red Fox

Raccoon

American Marten

Fisher

Ermine

Long-tailed Weasel

Weasel species

American Mink

American Badger

Striped Skunk

River Otter

Mountain Lion

Canada Lynx

Mule Deer

White-tailed Deer

Deer species

Moose

Elk

Pronghorn

American Bison

\section{TOTALS SEEN/HEARD}

ON COUNT DAY

TOTAL SPECIES SEEN/HEARD

TOTAL SPECIES

RECORDED BY TRACKS

TOTAL SPECIES

OTHERWISE RECORDED

SPECIES RECORDED

COUNT PERIOD

TOTAL SPECIES

COUNT PERIOD AND DAY

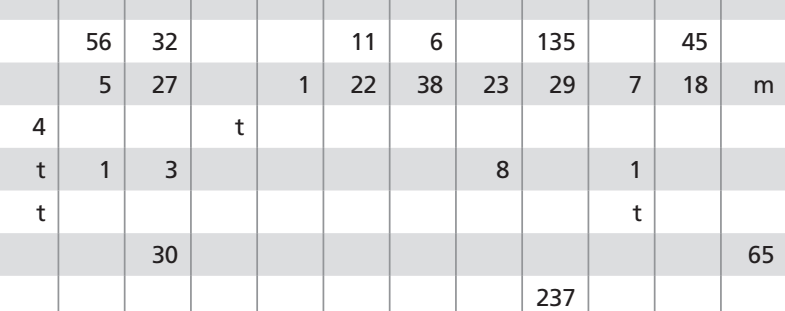

c
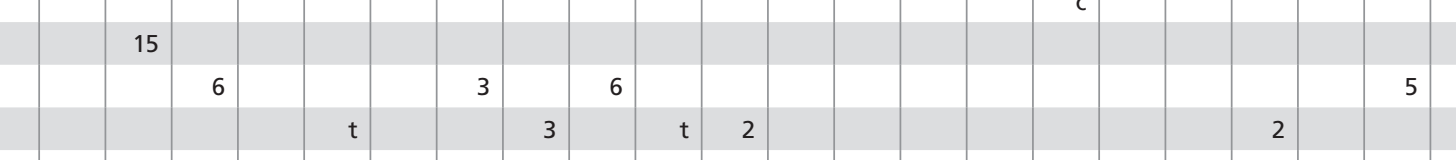

\begin{tabular}{|r|r|r|r|r|r|}
\hline $\mathrm{c}$ & $\mathrm{t}$ & 1 & & 1 & 2 \\
\hline
\end{tabular}

\begin{tabular}{|r|r|r|r|r|r|}
\hline $\mathrm{c}$ & & & $\mathrm{c}$ & & \\
\hline & $\mathrm{t}$ & 1 & & 1 & 2 \\
\hline
\end{tabular}

\begin{tabular}{|r|r|r|r|r|}
\hline & & & 3 \\
\hline
\end{tabular}

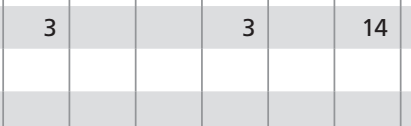

\begin{tabular}{|l|l|l|l|}
\hline & & & \\
\hline & & 7
\end{tabular}

\begin{tabular}{|r|r|r|r|r|r|}
\hline 13 & & 4 & 1 & 6 & \\
\hline & $\mathrm{L}$ & $\mathrm{L}$ & $\mathrm{L}$ & & \\
\hline & $\mathrm{L}$ & $\mathrm{L}$ & & & $\mathrm{L}$ \\
\hline & & $\mathrm{t}$ & & & \\
\hline
\end{tabular}

\begin{tabular}{l|l|l|l|l}
\hline & & & & \\
\hline & 1 & & L & L
\end{tabular}

\begin{tabular}{l|l}
\hline & L
\end{tabular}

t


Shrew species

Eastern Cottontail

Nuttall's Cottontail

Snowshoe Hare

White-tailed Jack Rabbit

Eastern Grey Squirrel

Eastern Fox Squirrel

American Red Squirrel

Northern Flying-Squirrel

Northern Pocket Gopher

American Beaver

Deer Mouse

Muskrat

Gapper's Red-Backed Vole

Meadow Vole

Vole species

House Mouse

Mouse species

American Porcupine

Coyote

Gray Wolf

Red Fox

Raccoon

American Marten

Fisher

Ermine

Long-tailed Weasel

Weasel species

American Mink

American Badger

Striped Skunk

River Otter

Mountain Lion

Canada Lynx

Mule Deer

White-tailed Deer

Deer species

Moose

Elk

Pronghorn

American Bison

TOTALS SEEN/HEARD

ON COUNT DAY

TOTAL SPECIES SEEN/HEARD

TOTAL SPECIES

RECORDED BY TRACKS

TOTAL SPECIES

OTHERWISE RECORDED

SPECIES RECORDED

COUNT PERIOD

TOTAL SPECIES

COUNT PERIOD AND DAY

\begin{tabular}{|r|r|r|r|r|} 
& 13 & & & \\
35 & 4 & 1 & $\mathrm{t}$ \\
& 7 & & \\
\end{tabular}

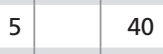

86

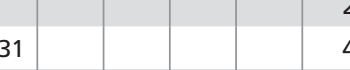

\begin{tabular}{|l|l|l|l|l|l|}
\hline 1 & 7 & 4 & & 8 \\
\hline
\end{tabular} \\ |}

政

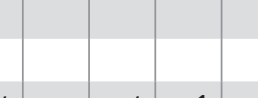

3

$\mathrm{t}$ \begin{tabular}{l|l|l|}
6 & $\mathrm{t}$ & $\mathrm{m}$
\end{tabular}

\begin{tabular}{|r|r|r|r|}
\hline $\mathrm{t}$ & & & 12 \\
\hline & & & 2 \\
\hline
\end{tabular}

\begin{tabular}{|r|r|}
\hline$t$ & $t$ \\
12 & $t$ \\
2 & \\
\hline 72 & 1 \\
\hline
\end{tabular}

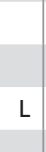

L

L

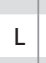

L
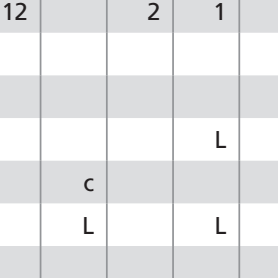

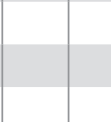




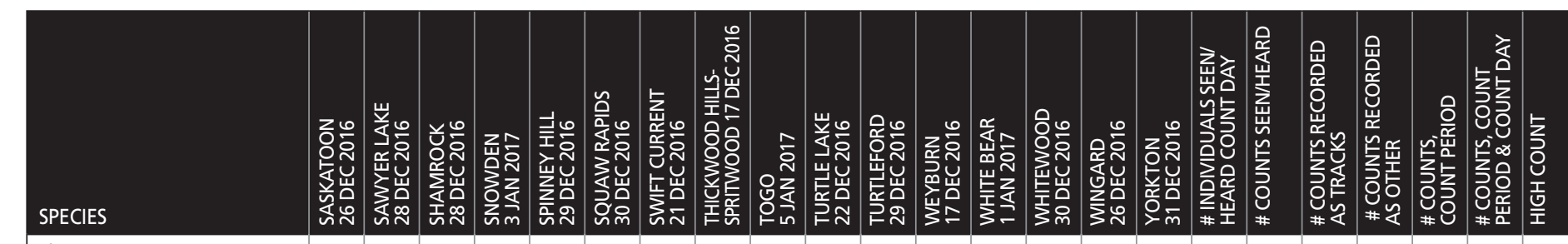

Shrew species

Eastern Cottontail

Nuttall's Cottontail

Snowshoe Hare

White-tailed Jack Rabbit

Eastern Grey Squirrel

Eastern Fox Squirrel

American Red Squirrel

Northern Flying-Squirrel

Northern Pocket Gopher

American Beaver

Deer Mouse

Muskrat

Gapper's Red-Backed Vole

Meadow Vole

Vole species

House Mouse

Mouse species

American Porcupine

Coyote

Gray Wolf

Red Fox

Raccoon

American Marten

Fisher

Ermine

Long-tailed Weasel

Weasel species

American Mink

American Badger

Striped Skunk

River Otter

Mountain Lion

Canada Lynx

Mule Deer

White-tailed Deer

Deer species

Moose

Elk

Pronghorn

American Bison

TOTALS SEEN/HEARD

ON COUNT DAY

TOTAL SPECIES SEEN/HEARD

TOTAL SPECIES

RECORDED BY TRACKS

TOTAL SPECIES

OTHERWISE RECORDED

SPECIES RECORDED

COUNT PERIOD

TOTAL SPECIES

COUNT PERIOD AND DAY

$t$

\begin{tabular}{r|r|r|r|r|r|r}
0 & 0 & 6 & 0 & 2 & 8 & 0 \\
\hline
\end{tabular} 